\title{
Negligência Infantil a Partir do Child Neglect Index Aplicado no Brasil
}

\section{Child Neglect Based on the Use of the Child Neglect Index Applied in Brazil}

\author{
Mara Silvia Pasian*, ${ }^{*}$, Marina Rezende Bazon $^{a}$, Sonia Regina Pasian ${ }^{a} \&$ Carl Lacharité ${ }^{b}$ \\ ${ }^{a}$ Universidade de São Paulo, Ribeirão Preto, SP, Brasil \\ $\&{ }^{b}$ Universidade de Quebec à Trois-Rivierés, Quebec, Canadá
}

\begin{abstract}
Resumo
Este estudo objetivou descrever a utilização do Child Neglect Index para identificar casos de crianças negligenciadas por seus pais/cuidadores no contexto brasileiro. Após adaptação inicial à realidade sociocultural brasileira, o instrumento foi aplicado em três grupos de indivíduos, cada qual com 30 díades de crianças/pais, totalizando 90 díades $(n=180)$, assim compostos: G1 (primeiro grupo) = casos oficialmente notificados por negligência infantil; G2 (segundo grupo) = casos suspeitos de negligência não notificados; G3 (terceiro grupo) = casos sem suspeita de negligência ou outra forma de maus-tratos (grupo de comparação); além de um quarto grupo de professoras dessas crianças $(n=11)$. Os resultados apontaram altos escores no índice de negligência em G1 e G2, e baixos escores em G3, sugerindo presença de indicadores de falta de supervisão parental referentes a cuidados com alimentação, vestimenta, higiene, saúde física, saúde mental e educação nos dois primeiros grupos avaliados, comparativamente às crianças sem suspeita de maus-tratos. Os atuais achados empíricos, embora preliminares em termos de adaptação do Child Neglect Index ao contexto do Brasil, foram promissores ao sinalizar potencial do instrumento como recurso para detecção/confirmação da negligência infantil, estimulando novas investigações e aprimoramento desse recurso técnico para favorecer adequada identificação e intervenção nesses casos.

Palavras-chave: Maus-tratos infantis, desenvolvimento da criança, relações pais-filhos, negligência infantil, avaliação psicológica.
\end{abstract}

\begin{abstract}
This study describes the use of Child Neglect Index to identify cases of children neglected by their parents/caregivers in the Brazilian context. After initial adaptation to the Brazilian sociocultural reality, the instrument was applied in three different groups. Each group is composed of 30 dyads of children/parents, totaling 90 dyads of participants $(n=180)$. They are: G1 (first group) = officially reported cases of child neglect; G2 (second group) = suspected cases of neglect, but not notified; G3 (third group) $=$ unsuspected cases of neglect or otherwise abuse (comparison group). There is also a fourth group composed of the children's teachers $(n=11)$. The results showed high scores on the index of neglect in G1 and G2, but low scores in G3, suggesting the presence of indicators of lack of parental supervision regarding the care of food, clothing, hygiene, physical health, mental health and education in the first two groups evaluated when compared to no suspicion of child maltreatment. The current empirical findings, although preliminary in terms of adaptation of Child Neglect Index to the Brazilian context, were promising to signal the potential of the instrument as a resource for detection/confirmation of child neglect. It stimulates further research and improvements in this technical resource to facilitate proper identification and intervention in these cases.

Keywords: Child abuse, child development, parent-child relationship, child neglect, psychological assessment.
\end{abstract}

Estima-se que a problemática dos maus-tratos seja significativa em todas as sociedades, gerando consequências negativas para o desenvolvimento de inúmeras crianças,

\footnotetext{
* Endereço para correspondência: Departamento de Psicologia, Faculdade de Filosofia, Ciências e Letras de Ribeirão Preto, Universidade de São Paulo, Av. Bandeirantes, 3900, Bloco 5, Monte Alegre, Ribeirão Preto, SP, Brasil 14040-901. E-mail: marasilvia123@yahoo. com.br, mbazon@ffclrp.usp.br, srpasian@ffclrp.usp.br e carl.lacharite@uqtr.ca
}

dispondo-se de evidências de que o número de casos conhecidos oficialmente é bem menor que o real (Bringiotti, 2000; Gonçalves, Ferreira, \& Marques, 2002; Smith, 2010). O reconhecimento e o enfrentamento do problema dos maus-tratos infantis e, dentre estes, da negligência, é desafio que implica na identificação de casos suspeitos, na notificação e na confirmação dos casos em instâncias oficiais que integram o sistema de proteção à infância (Lavergne \& Tourigny, 2000; C. B. G. Martins, 2010; Theodore, Runyan, \& Chang, 2007). 
As notificações sobre maus-tratos infantis são efetuadas aos órgãos oficiais, geralmente por meio de comunicações da comunidade em geral (vizinhos, familiares e parentes) ou de profissionais (professores, enfermeiros, médicos e policiais). No Brasil, é obrigatória a notificação de casos de maus-tratos conhecidos, suspeitos ou confirmados (Lei $\mathrm{n}^{\circ} 8.069,1990$ ), prevendo-se sanções para médicos, professores e responsáveis por estabelecimentos de Saúde e de Educação que deixem de fazê-lo (Lei no $8.069,1990)$. No entanto, persiste significativa taxa de subnotificação de casos de maus-tratos para com as crianças e os adolescentes (Ferreira, 2005; C. B. G. Martins, 2010; Mathews \& Bross, 2008; Theodore et al., 2007).

Entre as modalidades de maus-tratos infantis, a negligência infantil é a modalidade mais recorrente em diversos países, incluindo o Brasil (Bazon, 2008; Fallon et al., 2010; Ferreira, 2005; Hahm, Lee, Ozonoff, \& Van Wert, 2010; Lacharité, Éthier, \& Nolin, 2006; Mayer, Lavergne, Tourigny, \& Wright, 2007; Pasian, 2012; Roque \& Ferriani, 2007; Silvany, Siqueira, Rolim, \& Cavalcante, 2011; Slack, Holl, McDaniel, Yoo, \& Bolger, 2004; Theodore et al., 2007). Apesar da elevada incidência e de estar associada a consequências negativas em diversos aspectos do desenvolvimento (Corso \& Fertig, 2010; Mathews \& Bross, 2008; O'Donnell, Scott, \& Stanley, 2008; Pasian, 2012; Pires \& Miyazaki, 2005), a negligência infantil ainda tem sido pouco pesquisada na realidade brasileira.

Devido a dificuldades em sua definição e compreensão, muitos casos não são notificados pela comunidade leiga e/ou profissional e também são pouco valorizados nos próprios órgãos competentes, relacionados ao sistema de proteção infantil (Pasian, 2012). Conforme assinala F. F. S. Martins (2006), os profissionais que atuam no âmbito do sistema de proteção tendem a vivenciar grande dificuldade para conceituar negligência infantil, depreendendo-se falta de precisão teórica e de procedimentos. Na prática, esse contexto produz dificuldades na avaliação de possíveis casos, limitando também seu devido acompanhamento.

A temática da negligência impõe inúmeros desafios à investigação científica e à análise do problema, a começar pela ausência de uma definição consistente que dê conta de apreender a complexa realidade a que se refere (Calheiros \& Monteiro, 2000; Mayer et al., 2007; Smith, 2010). Ainda deve ser levado em conta que as concepções relativas a negligência são fortemente perpassadas por elementos da cultura, permanecendo sem consenso entre pesquisadores (Dubowitz, 2007; Dubowitz, Pitts, \& Black, 2004; Glaser, 2002; McSherry, 2007; Zuravin, 1999). Disso decorre uma dificuldade adicional, vivida tanto por pesquisadores como por profissionais, que é a de consubstanciar casos assinalados como "negligência", devido à heterogeneidade das situações associadas a essa modalidade de maus-tratos (Dubowitz, 2007; Mayer et al., 2007; McSherry, 2007; Smith, 2010).

Nesse contexto torna-se essencial delimitar o fenômeno em foco. A partir das considerações apresentadas pela World Health Organization e International Society for
Prevention of Child Abuse and Neglect (WHO \& ISPCAN, 2006), órgãos que desempenham papel relevante e normatizador na área, pode-se considerar que a negligência infantil se configura quando os pais, geralmente de um modo crônico, não têm vontade/disposição ou capacidades psicológicas requeridas para cuidar da criança e, dessa forma, acabam respondendo inadequadamente às necessidades de seus filhos e não demandam ou não conseguem aproveitar da ajuda de outras pessoas que poderiam/deveriam ajudar. Dessa forma, essas instituições caracterizam a negligência infantil como presente em contextos onde pais e/ou outros membros da família deixam de prover o desenvolvimento e o bem-estar da criança/adolescente (considerando que poderiam fazer isso), em uma das seguintes áreas: saúde, educação, desenvolvimento emocional, nutrição, abrigo e condições seguras. Essa delimitação conceitual da negligência infantil, compilada pelos autores a partir das referências anteriormente citadas, será adotada no presente trabalho.

Definida operacionalmente, a investigação do fenômeno da negligência infantil ainda circunda-se de outras dificuldades metodológicas, como a própria identificação dos casos. A escola é um contexto favorável para a detecção de casos suspeitos de maus-tratos, porque abrange grande percentual da população infantil. Nas escolas, as crianças passam muitas horas por dia e muitos dias por ano, convivendo com professores e outros profissionais, o que facilita a apreensão de sinais/indicadores de maus-tratos, incluindo os de negligência, que servem de alerta à identificação de casos (Bazon, 2006; Lavergne \& Tourigny, 2000). Ademais, sabe-se que os casos suspeitos de maus-tratos, na Educação, tendem a ser confirmados (Smith, 2010).

No entanto, a subnotificação dos casos de negligência infantil no sistema educacional também é significativa (Smith, 2010), estimando-se que a cifra dessa problemática seja ainda maior nas séries iniciais da escolarização (Bazon, 2006; Silva \& Ferriani, 2007). Um fator claramente reconhecido na tomada de decisão sobre notificar ou não um caso suspeito de maus-tratos (e em especial de negligência infantil) às autoridades, no âmbito do setor educacional, é o medo do envolvimento pessoal com a problemática, temendo-se represálias por parte dos envolvidos (Granville-Garcia, Souza, Menezes, Barbosa, \& Cavalcanti, 2009; Silva \& Ferriani, 2007).

Considera-se que, em meio às razões pelas quais há subnotificação e/ou menor preocupação para com o acompanhamento dos casos de negligência infantil, destaca-se a falta de instrumentos adequados para avaliação das crianças e de seus cuidadores, no contexto do Brasil. O levantamento bibliográfico da literatura científica nacional a respeito do tema aponta reduzidos recursos instrumentais para essa prática. Um dos poucos recursos sistematicamente examinados é o Child Abuse Potential Inventory-CAP, instrumento que detecta o potencial de risco, em cuidadores de crianças e adolescentes, para os maus-tratos (Bérgamo, 2007; Bérgamo, Pasian, Ávila de Mello, \& Bazon, 2009; Rios, 2010; Rios, Williams, Schelini, Bazon, \& Piñón, 
2013). O objetivo principal do instrumento é o de avaliar fatores de risco de natureza psicológica e psicorrelacional, especificamente associados aos maus-tratos físicos e à negligência em adultos responsáveis por crianças. Bérgamo et al. (2009) avaliaram sua fidedignidade (consistência interna), chegando a estimativas adequadas para a escala de Abuso (0,92 e 0,93 para grupos estudados). Rios (2010), com vistas à adaptação transcultural do CAP para o Brasil, verificou que a versão em português apresenta adequada equivalência semântica e demonstrou a validade de construto do instrumento, figurando como um dos poucos recursos técnicos disponíveis nessa área no Brasil. Desse modo, tanto a pesquisa quanto a prática em situações de risco ao desenvolvimento infantil ganhariam contribuições significativas com a pesquisa de instrumentos adicionais para avaliação dos maus-tratos infantis, em especial da negligência, foco do presente trabalho.

O panorama apresentado sugere que investigações sejam feitas na procura de recursos técnicos e instrumentos profissionais que facilitem a identificação e a confirmação de casos suspeitos de negligência infantil. O desenvolvimento de estratégias e de procedimentos facilitadores da identificação de casos de crianças negligenciadas pelos órgãos competentes e também pelos profissionais que com elas trabalham, sobretudo nos setores da Saúde e Educação, poderá favorecer o conhecimento e a devida caracterização do fenômeno em questão, permitindo elaboração de intervenções adequadas nessa área.

Diante desse contexto, o presente trabalho objetivou descrever a utilização do Child Neglect Index para identificar casos de crianças negligenciadas por seus pais/ cuidadores no contexto brasileiro. Esse instrumento de avaliação foi elaborado por Trocmé (1996) no Canadá, com a finalidade de detectar e confirmar casos de negligência infantil. Foi originalmente desenvolvido em inglês e possui uma versão validada em francês (Brousseau, 2000). Caracteriza-se como inventário do tipo checklist, por meio do qual um avaliador (no caso, o pesquisador), afere e pontua um escore (de zero até 60 pontos) para a presença de comportamentos parentais sistematizados em seis possíveis categorias, relativas aos seguintes campos de atenção: supervisão parental global, cuidados com a alimentação/nutrição, cuidados com vestimenta adequada e higiene, cuidados com a saúde física, cuidados com a saúde mental, educação e desenvolvimento da criança. Resulta num índice final que, quanto maior, indicará sinais mais consistentes de negligência infantil.

Esse instrumento avaliativo foi revisto e aprimorado por Brousseau (2000), em versão em francês, a qual tem sido recorrentemente utilizada em recentes estudos desenvolvidos em Quebec/Canadá para caracterização de crianças negligenciadas. Essa versão do Child Neglect Index mostrou-se adequada a seus objetivos, com adequados indicadores psicométricos, como apontaram Nolin e Turgeon (2004), Strauss e Kantor (2005), além de Sullivan (2000). Esse instrumento caracteriza-se, portanto, como promissor para a pesquisa e a atuação de profissionais que atuam nos órgãos de proteção à criança e ao adolescente para detecção e confirmação da negligência infantil, constituindo-se no objeto da presente investigação científica.

\section{Método}

Trata-se de estudo transversal, descritivo e comparativo de grupos, de natureza exploratória, caracterizando achados do Child Neglect Index para identificar casos de crianças negligenciadas por seus pais/cuidadores no contexto do Brasil.

\section{Participantes}

A presente investigação foi realizada com três grupos de indivíduos, cada qual composto por 30 díades de crianças/pais, totalizando 90 díades de participantes $(n=180)$, além de um quarto grupo de professoras dessas crianças $(n=11)$. As crianças cursavam as séries escolares iniciais do ensino fundamental (segundo e terceiros anos), com idade entre seis e oito anos, estudantes de escolas públicas (uma escola municipal e cinco escolas estaduais) de uma cidade do interior do Estado de São Paulo e foram devidamente autorizadas para participar do estudo por seus pais/responsáveis. Todas as crianças foram selecionadas nas mesmas escolas, de modo a se garantir semelhantes condições socioculturais entre os participantes, permitindo posterior comparação dos resultados.

Trata-se, no entanto, de amostra de conveniência, respeitando-se os critérios de seleção aqui referidos. $\mathrm{O}$ primeiro grupo (G1) foi composto por casos oficialmente notificados por negligência infantil ao Conselho Tutelar da cidade, incluindo as 30 crianças e seus respectivos responsáveis, totalizando 30 díades. $\mathrm{O}$ segundo grupo (G2) foi formado por casos suspeitos de negligência, tendo por base observações de professores $(n=11)$ no contexto escolar, porém não notificados ao Conselho Tutelar (portanto, desconhecidos no sistema oficial de proteção). Esse grupo também foi composto por 30 crianças e seus respectivos cuidadores/responsáveis legais, no número de 30 participantes, compondo trinta díades. $\mathrm{O}$ terceiro grupo (G3) foi constituído por 30 crianças sem suspeita de negligência ou outra forma de maus-tratos (grupo de comparação), tendo por base observações de professores $(n=11)$ no contexto escolar, e também seus respectivos pais ou responsáveis legais, totalizando 30 díades. Assim, o presente estudo contou com a participação de 90 crianças e 90 pais/cuidadores, além das 11 professoras (docentes de crianças de G1 e referência para identificação dos casos para comporem G2 e G3, buscando-se levantar informações padronizadas referentes ao desenvolvimento das crianças dos três grupos).

Os pais/cuidadores que participaram do estudo foram aqueles oficialmente registrados nas escolas como responsáveis legais pelas crianças, sendo majoritariamente do sexo feminino (mãe, tia ou avó da criança). Por sua vez, os professores envolvidos no estudo foram aqueles das escolas colaboradoras que aceitaram participar do estudo, 
após ter sido realizada uma palestra em seu ambiente escolar sobre o tema "maus-tratos infantis" e sobre o projeto de pesquisa em si, convidando-os a colaborar. Compõem, portanto, um subgrupo de voluntários selecionados apenas por viabilidade prática, a partir de seu livre consentimento com essa pesquisa.

\section{Instrumentos}

Questionário de Caracterização Sócio-Demográfica. Elaborado com base no modelo proposto por Bringiotti (1999), coleta informações do respondente (cuidador ou responsável pela criança) em termos de idade, sexo, nível educacional, estado civil, número de filhos ou crianças sob seus cuidados, além de dados referentes a condições de vida, como situação empregatícia, número de moradores na mesma residência, número de cômodos da casa e impressão do bairro de residência (Bazon, Mello, Bérgamo, \& Faleiros, 2010).

Inventário de Potencial de Risco para os Maus-Tratos Infantis (CAP). Construído por Milner (1986), foi traduzido e adequado semanticamente para o português do Brasil por membros do GEPDIP (Grupo de Estudos e Pesquisa em Desenvolvimento e Intervenção Psicossocial - Universidade de São Paulo [USP]) e do LAPREV (Laboratório de Prevenção da Violência - Universidade Federal de São Carlos [UFSCar]). Na sequência, o instrumento foi alvo de estudos na realidade brasileira (Bérgamo, 2007; Bérgamo et al., 2009; Rios, 2010; Rios et al., 2013), tendo-se atestado suas qualidades psicométricas. O inventário é constituído por 160 itens, subdivididos em escalas específicas, aos quais a pessoa concorda ou discorda da afirmação. A Escala de Abuso é a principal do CAP, sendo composta por 77 itens, constituindo-se no instrumento utilizado no presente estudo, conforme padronização de Bérgamo (2007).

Child Neglect Index. Elaborado por Trocmé (1996) no Canadá, com a finalidade de detectar e confirmar casos de negligência infantil. A versão em francês do instrumento (Brousseau, 2000) tem sido a mais utilizada em pesquisas, mostrando-se adequada a seus objetivos no contexto do Canadá (Nolin \& Turgeon, 2004; Strauss \& Kantor, 2005; Sullivan, 2000; Trocmé, 1996), correspondendo ao instrumento usado no presente trabalho. Ele foi devidamente traduzido para o português pela primeira autora deste trabalho, com posterior revisão desta tradução por pesquisador da área com fluência em português e francês, chegando-se a uma versão de consenso. Esta versão em português, por sua vez, foi encaminhada, juntamente com sua retroversão em francês, para análise técnica da equipe elaboradora do Child Neglect Index, efetivando exame da adequação técnica e de conteúdo do instrumento, tendo sido aprovada (C. Lacharité, comunicação pessoal, 27 de maio, 2009) e autorizada para uso em pesquisa no Brasil e no presente trabalho (N. Trocmé, comunicação pessoal, 27de maio, 2009). Trata-se, portanto, de um instrumento que foi traduzido para o português do Brasil, mas que ainda precisa ser validado, compondo-se, nesse momento, estudo exploratório a seu respeito.
$\mathrm{O}$ instrumento organiza-se como uma listagem de comportamentos (checklist) do cuidador/responsável pela criança, sendo os itens presentes ou ausentes assinalados por um observador externo (o pesquisador). A partir do conjunto desses indicadores atribui-se um escore (de zero até 60 pontos) para a presença de seis tipos de cuidados: supervisão parental global; alimentação/nutrição; vestimenta adequada e higiene; saúde física; saúde mental; educação e desenvolvimento da criança. A seguir é identificada a categoria avaliativa com escore mais elevado, que corresponderá à nota final do índice de negligência do Child Neglect Index. Quanto maior esse índice final, os sinais de negligência para com crianças/adolescentes também serão mais consistentes. Cabe destacar, ainda sobre o sistema de codificação dos resultados, que pontos são adicionados ao escore final da soma das seis categorias de negligência a partir da idade da criança. Quanto menor a idade da criança, maior será essa pontuação adicional, correspondendo ao seguinte esquema: 20 pontos para idades entre zero e dois anos, 15 pontos para idades entre três e cinco anos; cinco pontos para idades entre seis e 12 anos. Acima desta idade não são acrescidos pontos adicionais aos escores obtidos pelo instrumento. No caso das crianças desse estudo (entre seis e oito anos), foram adicionados cinco pontos ao índice final observado pelo instrumento.

A padronização do Child Neglect Index (Brousseau, 2000), seguida no presente trabalho, prevê que, para o devido preenchimento, o avaliador deve realizar visitas domiciliares (duas a três vezes na mesma moradia, sem fixação de período, com duração média entre 30 e 60 minutos), coletando informações relativas aos itens focalizados no checklist do instrumento. Também devem ser procurados os professores dessas crianças em seu próprio ambiente escolar, de modo a permitir a busca ativa de informações relativas à rotina de cuidados a que estão submetidas. Somente de posse do conjunto desses dados é que o avaliador poderá preencher, com a devida segurança, os dados do instrumento em si, permitindo sua classificação de resultados.

Para a interpretação e análise dos resultados do Child Neglect Index foram utilizados os padrões originais produzidos no Canadá (Brousseau, 2000), por não existirem referenciais disponíveis no Brasil até o momento. Desse modo, os índices finais no instrumento foram assim classificados: (a) escore entre zero e 20 pontos: ausência de negligência, o que indica que as necessidades da criança estão atendidas de modo satisfatório; (b) escore entre 25 e 45 pontos: negligência leve, sugestiva de alguma necessidade de intervenção profissional com os cuidadores desta criança; (c) escore maior que 50: negligência moderada ou severa, descrevendo um quadro que necessita de intervenção profissional para proteção da criança.

\section{Procedimentos}

Nesse estudo foram tomadas as medidas necessárias para a devida observação dos princípios éticos que regem a pesquisa com seres humanos, de acordo com as 
diretrizes e normas vigentes. Primeiramente, a pesquisa recebeu aprovação da Secretaria Municipal de Educação e dos Conselheiros Tutelares da cidade, com posterior apreciação e aprovação pelo Comitê de Ética em Pesquisa da Faculdade de Filosofia, Ciências e Letras de Ribeirão Preto (FFCLRP, parecer $n^{\circ}$. 394/2008) - USP. Após esclarecimentos e convite a possíveis voluntários do estudo, conforme critérios de seleção de participantes, aqueles que concordaram com a proposta assinaram o respectivo Termo de Consentimento Livre e Esclarecido (pais/responsáveis ou cuidadores e professores das crianças), autorizando o estudo e suas atividades. Suas crianças também foram convidadas ao trabalho e apenas participaram aquelas que aceitaram, livremente, colaborar com as atividades.

Os dados do presente trabalho foram coletados pela primeira autora, no período entre 2009 e 2011, respeitando as diretrizes técnicas padronizadas de cada instrumento aqui utilizado. A Escala de Abuso do CAP foi aplicada e avaliada conforme referenciais de Bérgamo (2007) e Bérgamo et al. (2009). O Child Neglect Index foi preenchido com base em condições observadas na casa dos pais/cuidadores das crianças selecionadas, a partir das informações apontadas por esses informantes e por relatos dos professores dessas crianças. Para tanto, a pesquisadora seguiu o procedimento padrão do instrumento (Brousseau, 2000), ou seja, realizou duas a três visitas domiciliares aos 90 pais/cuidadores participantes da pesquisa, observando e coletando informações sobre as crianças. A mesma pesquisadora visitou e entrevistou os professores das 90 crianças participantes da pesquisa, possuindo adequadas condições técnicas para o correto preenchimento do instrumento. A pesquisadora, então, atribuiu um valor (na escala de zero a $60)$ a cada categoria avaliativa proposta pelo instrumento, representativa da realidade dos cuidados destinados às crianças participantes do projeto. Visto estarem todas entre seis e oito anos, tiveram cinco pontos acrescidos no escore final em cada categoria do instrumento, sendo, por fim, creditado e classificado o maior valor identificado pelo Child Neglect Index, como preconiza seu padrão de análise.

Os resultados foram sistematizados em planilhas do programa Excel da Microsoft e posteriormente examinados com auxílio do Statistical Package of Social Sciences (SPSS, versão 16.0), de modo descritivo e inferencial, visando atender aos objetivos propostos no estudo. As análises iniciais foram descritivas, de modo a caracterizar cada um dos grupos aqui estudados em termos sociodemográficos e no índice de negligência. A seguir, foram realizadas comparações estatísticas inferenciais dos resultados entre os três grupos avaliados, recorrendo-se a análises de variância (ANOVA e Pós-teste de Duncan) ou ao Teste Exato de Fisher, conforme a variável focalizada. Para todas essas análises foi considerado o nível de significância menor ou igual a 0,05 .

\section{Resultados}

No presente trabalho um dos cuidados metodológicos foi a composição de grupos de participantes com características sociodemográficas semelhantes. Assim, as crianças selecionadas viviam nos mesmos bairros e estudavam nas mesmas escolas, apesar de suas especificidades históricas e de contextos de vida. Foi possível, a partir do questionário sociodemográfico respondido por pais/cuidadores, caracterizar suas famílias e, mais particularmente, o adulto responsável. A Tabela 1 apresenta, de forma sintética, as principais variáveis caracterizadoras do conjunto de pais ou cuidadores que participaram do estudo, informando sobre suas respectivas crianças.

Tabela 1

Informações Sociodemográficas das Famílias Estudadas

\begin{tabular}{|c|c|c|c|c|c|c|c|c|c|}
\hline \multirow{2}{*}{\multicolumn{2}{|c|}{$\begin{array}{c}\text { Grupos } \\
\text { Variável } \\
\text { (relativa ao respondente) } \\
\end{array}$}} & \multicolumn{2}{|c|}{$\begin{array}{l}\text { Grupo 1 } \\
(n=30)\end{array}$} & \multicolumn{2}{|c|}{$\begin{array}{l}\text { Grupo } 2 \\
(n=30)\end{array}$} & \multicolumn{2}{|c|}{$\begin{array}{l}\text { Grupo } 3 \\
(n=30)\end{array}$} & \multirow{2}{*}{ Estatística } & \multirow{2}{*}{$p$} \\
\hline & & Média & $D P$ & Média & $D P$ & Média & $D P$ & & \\
\hline \multicolumn{2}{|l|}{ Idade (em anos) } & 34,47 & 11,34 & 33,67 & 9,90 & 33,43 & 8,10 & $F_{(2,87)}=0,09$ & 0,914 \\
\hline \multicolumn{2}{|l|}{ Anos de estudo } & 4,53 & 1,43 & 5,07 & 2,77 & 9,43 & 3,65 & $F_{(2,87)}=28,29$ & $<0,001$ \\
\hline \multicolumn{2}{|c|}{ Renda familiar (em reais)* } & 743,33 & 225,42 & 905,00 & 368,21 & $1.323,33$ & 806,30 & $F_{(2,87)}=9,64$ & $<0,001$ \\
\hline \multicolumn{2}{|c|}{ Idade (ao ter primeiro filho) } & 17,40 & 2,06 & 18,90 & 2,71 & 21,57 & 2,13 & $F_{(2,87)}=24,88$ & $<0,001$ \\
\hline \multirow{3}{*}{ Sexo $* *$} & Feminino & \multicolumn{2}{|c|}{29} & \multicolumn{2}{|c|}{26} & \multicolumn{2}{|c|}{29} & Teste Exato & 0,357 \\
\hline & Masculino & \multicolumn{2}{|c|}{01} & \multicolumn{2}{|c|}{04} & \multicolumn{2}{|c|}{01} & de Fisher & - \\
\hline & Própria & \multicolumn{2}{|c|}{10} & \multicolumn{2}{|c|}{13} & \multicolumn{2}{|c|}{12} & & 0,797 \\
\hline \multirow{2}{*}{ Habitação** } & Alugada & \multicolumn{2}{|c|}{13} & \multicolumn{2}{|c|}{13} & \multicolumn{2}{|c|}{16} & $\begin{array}{l}\text { Teste Exato } \\
\text { de Fisher }\end{array}$ & 0,773 \\
\hline & Cedida & \multicolumn{2}{|c|}{07} & \multicolumn{2}{|c|}{04} & \multicolumn{2}{|c|}{02} & & 0,217 \\
\hline \multicolumn{2}{|c|}{ Planejamento gravidez $* *$} & \multicolumn{2}{|c|}{02} & \multicolumn{2}{|c|}{08} & \multicolumn{2}{|c|}{12} & $\begin{array}{c}\text { Teste Exato } \\
\text { de Fisher }\end{array}$ & 0,007 \\
\hline
\end{tabular}

Notas. * Salário mínimo vigente na época de coleta de dados: R \$ 540,00. ** Nas variáveis "Sexo", "Habilitação" e "Planejamento da gravidez" o dado corresponde ao número de casos identificados em cada categoria aqui considerada. 
Pasian, M. S., Bazon, M. R., Pasian, S. R. \& Lacharité, C. (2015). Negligência Infantil a Partir do Child Neglect Index Aplicado no Brasil.

Em termos gerais, nota-se que os cuidadores que participaram do estudo foram majoritariamente do sexo feminino, especificamente mãe, tia ou avó da criança, as quais constavam, nas respectivas escolas, como legalmente responsáveis pelas crianças avaliadas no trabalho. Também não emergiram diferenças entre os três grupos avaliados no tocante ao tipo de moradia, sendo que a maioria habitava em casa alugada ou cedida $(67,0 \% \mathrm{em} \mathrm{G1}, 56,0 \% \mathrm{em} \mathrm{G} 2 \mathrm{e}$ $60,0 \%$ em G3). Os grupos também não diferiram em termos da idade média do cuidador responsável. Em termos gerais, portanto, os três grupos de díades mostraram-se similares, permitindo análises comparativas de seus resultados.

Cabe destacar, no entanto, que em termos de escolaridade, G3 apresentou número médio de anos de estudo $(9,4$ anos de escolaridade) significativamente superior a G1 e G2 (respectivamente 4,5 e 5,1 anos de estudo). A diferença também foi identificada em relação à renda familiar: no grupo de referência (G3) os cuidadores referiram viver com "mais de dois até três salários", enquanto G1 e G2 relataram receber, em média, renda "mais de um até dois salários".
O questionário também revelou alguns fatores de risco para a ocorrência de maus-tratos infantis em G1 e G2, segundo evidências de Bérgamo (2007) e Rios (2010). A partir das considerações desses referidos trabalhos, além do nível de escolaridade e da renda familiar, os grupos G1 e G2 do presente estudo apresentaram maior índice de mães com idade precoce ao ter o primeiro filho (com menos de 20 anos) e o não planejamento da gravidez, variáveis também apontadas como fatores de risco para maus-tratos por Bringiotti (2000). Com base nesses dados gerais, pode-se considerar que as características sociodemográficas das díades avaliadas permitiram indicadores de algumas similaridades socioambientais dos participantes, embora também com marcadores que exigirão adequada interpretação no conjunto de dados presentemente obtidos.

Por sua vez, os resultados da Escala de Abuso do Inventário de Potencial de Maus-tratos Infantis (CAP) estão sintetizados na Tabela 2. Procurou-se verificar a existência ou não de diferenças entre os grupos aqui considerados.

Tabela 2

Resultados Médios e Comparação Estatística dos Grupos na Escala de Abuso do Inventário de Potencial de Maustratos Infantis (CAP)

\begin{tabular}{cccccccc}
\hline Grupo & $n$ & Média & $D P$ & Mínimo & Máximo & ANOVA & $p$ \\
\hline 1 & 30 & 358,7 & 59,2 & 258 & 448 & & \\
2 & 30 & 317,9 & 99,6 & 87 & 445 & $F_{(2,87)}=34,33$ & $\leq 0,001$ \\
3 & 30 & $186,7^{*}$ & 88,0 & 36 & 396 & & \\
\hline
\end{tabular}

Nota. ${ }^{*} \mathrm{G} 3$ apresentou média na Escala de Abuso do CAP estatisticamente inferior a G1 e G2, considerados similares (pós-teste de Duncan, $\operatorname{com} p \leq 0,001)$.

Pelo exposto na Tabela 2, observa-se que há diferença estatisticamente significativa entre os grupos quanto ao escore médio obtido na Escala de Abuso do CAP. O grupo notificado por negligência (G1) teria um potencial de abuso semelhante a G2 (suspeitos de negligência, porém não notificados), porém ambos significativamente diferentes de G3 (grupo de referência), o qual apresentou resultados sugestivos de menor potencial de abuso infantil por parte dos cuidadores. O pós-teste de Duncan mostrou que G3 alcançou uma média significativamente inferior dos demais grupos, considerados homogêneos.

Por fim, foram analisados os dados referentes ao Child Neglect Index para verificar a existência ou não de diferenças entre os grupos examinados, no tocante à presença de indicadores relacionados à supervisão parental, cuidados físicos (alimentação, vestimenta, higiene), saúde física e saúde mental, bem como com a educação e o desenvolvimento da criança. Esses resultados compõem a Tabela 3.

Tabela 3

Resultados Médios e Comparação Estatística dos Grupos no Child Neglect Index

\begin{tabular}{cccccccc}
\hline GRUPO & $n$ & Média & $D P$ & Mínimo & Máximo & ANOVA & $p$ \\
\hline 1 & 30 & 48,3 & 13,9 & 30 & 65 & & \\
2 & 30 & 45,2 & 12,2 & 30 & 65 & $F_{(2,87)}=147,06$ & $<0,001$ \\
3 & 30 & $2,5^{*}$ & 7,6 & 0 & 25 & & \\
\hline
\end{tabular}

Nota. ${ }^{*} \mathrm{G} 3$ apresentou média no Child Neglect Index estatisticamente inferior a G1 e G2, considerados similares (pós-teste de Duncan, $\operatorname{com} p \leq 0,001)$. 
Pode-se identificar diferença estatisticamente significativa entre as médias dos grupos no que diz respeito ao índice de negligência. Mais uma vez, G1 apresentou índice similar ao G2, revelando que as crianças desses dois grupos vivem mais recorrentemente situações que podem caracterizar negligência, diferentemente de G3 (grupo de referência), para o qual o índice de ocorrência dessas situações foi significativamente menor. Os escores obtidos no grupo de crianças notificadas no Conselho Tutelar (G1), como as crianças de G2 (indicadas pelos seus respectivos professores) apresentaram escores médios entre 48 e 45 pontos, respectivamente. Revelaram, assim, com a utilização do instrumento Child Neglect Index, que essas crianças tendem a vivenciar situações de negligência por parte de seus cuidadores, sugestivo de alguma necessidade de intervenção profissional com esses indivíduos.

Cabe destacar ainda que, em G1, a maioria dos casos (em 20 díades) apresentou escore igual ou maior que 50, entre os quais oito crianças apresentaram escore de 65, sinalizando negligência moderada ou severa, descrevendo um quadro que necessita de intervenção profissional para proteção da criança. Em G2 também a maioria dos casos (em 19 díades) apresentou escore igual ou maior que 50 (três com escore maior que 60). Isso reafirma que tanto as crianças com notificações no conselho tutelar (G1), como as indicadas pelos professores como supostamente negligenciadas (G2), apresentaram índices considerados preocupantes em relação à negligência por parte de seus cuidadores. Por fim, faz-se claro o contraste com G3, constituído como grupo de referência do estudo, onde apenas três casos atingiram escore 25 no Child Neglect Index, confirmando suas possibilidades informativas no contexto do Brasil. As demais 27 crianças de G3 obtiveram escore igual a zero nesse instrumento, portanto consideradas sem suspeita de negligência, corroborando a indicação realizada por seus respectivos professores.

\section{Discussão}

O presente estudo objetivou descrever a utilização do Child Neglect Index para identificar casos de crianças negligenciadas por seus pais/cuidadores no contexto brasileiro. Esse recurso de avaliação psicológica é utilizado em diferentes países e auxilia vários segmentos profissionais na identificação de crianças negligenciadas (Nolin \& Turgeon, 2004; Strauss \& Kantor, 2005; Sullivan, 2000; Trocmé, 1996).

No tocante ao potencial de risco de abuso dos cuidadores, avaliado com a aplicação do instrumento CAP e por meio dos indicadores de negligência, obtidos com a aplicação do Child Neglect Index, os resultados confirmaram os dados clínicos existentes sobre os casos, evidenciando indicadores de validade desses dois instrumentos de avaliação psicológica no contexto do Brasil. Os dados apontaram resultados similares em G1 (casos de negligência infantil notificados nos Conselhos Tutelares) e em G2 (crianças indicadas pelos professores como sendo suspeitas de sofrerem negligência). Nesses dois grupos, a Escala de Abuso do CAP atingiu escores médios muito altos (359 pontos em G1 e 318 pontos em G2). Segundo estudos realizados, o escore igual ou acima de 215 é considerado risco elevado para abuso e negligência (Bérgamo et al., 2009; Rios, 2010). Os atuais achados, bastante superiores a esta nota de corte sugerida, indicam que nos grupos clínicos presentemente avaliados (G1 e G2), os cuidadores responsáveis evidenciaram características psicológicas e psicorrelacionais que constituem risco para maus-tratos dirigidos a essas crianças.

De forma exploratória, esses resultados podem ser analisados com base na nota de corte (correspondente a 214 pontos), obtida para o CAP na padronização do instrumento para a população dos Estados Unidos. Tomando essa referência, pode-se dizer que em G1 e em G2 os cuidadores participantes apresentaram um alto índice de potencial para o cometimento dos maus-tratos, diferentemente de G3. No Brasil, os estudos do CAP realizados com pais/ cuidadores também notificados aos órgãos de proteção infantil, por abuso físico (Bérgamo, 2007) e negligência (Ávila de Mello, 2008), mostraram escores médios totais na Escala de Abuso, respectivamente, 223,87 (DP $\pm 99,30)$ e $258,37(D P \pm 97,73)$. Embora superiores à nota de corte americana (214), são inferiores às médias de G1 e de G2 aqui investigados. Em contraposição, no grupo de referência (G3), a média de pontos na Escala de Abuso do CAP atingiu 187 pontos, valor estatisticamente diferente dos grupos clínicos e abaixo da nota de corte americana (214 pontos), o que indicaria baixo potencial de risco para os maus-tratos (Bérgamo et al., 2009; Rios, 2010).

$\mathrm{Na}$ mesma direção, os resultados dos indicadores de negligência obtidos com a aplicação do Child Neglect Index apontaram similaridade entre G1 e G2, com clara diferenciação de G3. As crianças de G1 e de G2 pareceram vivenciar, mais recorrentemente, situações que podem caracterizar negligência, diferentemente de G3 (grupo de referência), para o qual o índice de ocorrência dessas situações foi significativamente menor. Os altos escores obtidos por G1 e G2 nesse instrumento apontaram a presença de indicadores relacionados à falta de supervisão parental referente a cuidados com alimentação, vestimenta e higiene, com saúde física e mental das crianças, bem como com sua educação e seu desenvolvimento. De acordo com vários autores (Lacharité et al., 2006; Reppold, Pacheco, Bardagi, \& Hutz, 2002) esses indicadores podem ser considerados sugestivos de negligência para com as crianças.

Os dados obtidos mostraram que o instrumento Child Neglect Index foi bastante sensível na caracterização/ confirmação dos casos de negligência para a realidade brasileira, na medida em que, nos dois grupos de crianças que apontaram suspeitas de negligência o instrumento mostrou índices altos (G1 e G2), estatisticamente significativos. Revelou, portanto, indicadores de vivência da negligência pelas crianças, diferentemente do grupo de referência (G3), onde o índice não foi sugestivo de experiências de negligência infantil. 
Pasian, M. S., Bazon, M. R., Pasian, S. R. \& Lacharité, C. (2015). Negligência Infantil a Partir do Child Neglect Index Aplicado no Brasil.

Em suma, o conjunto dos resultados atinentes aos indicadores de negligência presentemente encontrados pode ser considerado promissor enquanto evidências para o uso do Child Neglect Index como um instrumento útil na prática cotidiana de profissionais responsáveis por crianças, como no ambiente escolar. A sistematização de informações e seu aprimoramento técnico enquanto instrumento de avaliação psicológica poderá favorecer a adequada identificação e posterior intervenção com os casos de crianças em situação de risco ao desenvolvimento, como nos quadros de negligência infantil.

Sabe-se que considerável proporção de reais casos de negligência infantil não é notificada por ser considerada de "pouca gravidade" por muitos profissionais e mesmo cuidadores (F. F. S. Martins, 2006). No geral, refere-se a modalidades de maus-tratos que não deixam marcas físicas no corpo da criança ou qualquer outro tipo de sequela dessa natureza, o que pode ser erroneamente interpretado como sinalizador de reduzido impacto no desenvolvimento infantil. Nesse sentido, é importante sublinhar as considerações realizadas no estudo de Gracia (1995), cujo objetivo envolveu análise comparativa das consequências negativas ao desenvolvimento infantil em função da gravidade da negligência. Os resultados dessa investigação demonstraram que os prejuízos para as crianças foram equiparáveis entre casos considerados "não tão graves" e casos "muito graves", ou seja, igualmente preocupantes no tocante ao número e à natureza dos problemas no desenvolvimento.

Pessoas leigas e muitos profissionais acreditam que a negligência não provoca consequências importantes ao desenvolvimento da criança, porque ela é caracterizada mais propriamente pela omissão de comportamentos por parte dos pais/cuidadores, diferentemente das outras formas de maus-tratos (F. F. S. Martins, 2006; Nolin \& Turgeon, 2004; Pasian, 2012). A identificação e a caracterização da negligência se tornam, portanto, complexas, sendo muitas vezes mal compreendida, até mesmo pelos profissionais dos órgãos de proteção, responsáveis pelo bem estar das crianças e adolescentes (Bazon, 2008), o que torna evidente a necessidade de adequados instrumentos para sua avaliação, como o proposto nesse estudo.

Profissionais da área da Saúde e Educação podem contribuir de forma efetiva para a intervenção em casos de maus-tratos infantis, fazendo as notificações e estando cientes da obrigatoriedade desse ato, para que possam ser tomadas as medidas necessárias aos casos. No entanto, para que isso ocorra de forma efetiva, é necessário que esses profissionais recebam informações e tenham ciência das consequências advindas da negligência para com crianças, permitindo, inclusive, o adequado reconhecimento dos fatores de risco associados ao quadro, como argumentado por Bazon (2008). Com isso, medidas preventivas e intervenções para com os pais/cuidadores poderiam ser ofertadas, de modo a beneficiar as crianças que se encontram vulneráveis, evitando ou amenizando consequências negativas ao seu desenvolvimento.

\section{Considerações Finais}

A complexidade conceitual e de caracterização operacional da negligência infantil dificultam o adequado reconhecimento e valorização desse tipo de maus-tratos, sabidamente lesivo ao desenvolvimento de crianças e adolescentes, como apontado pela literatura científica da área. São claras as evidências de que o fenômeno da subnotificação dos maus-tratos infantis é um problema social significativo, sublinhado em diversos estudos brasileiros e internacionais (Bazon, 2006; Bringiotti, 2000; Gonçalves et al., 2002; C. B. G. Martins, 2010; Mathews \& Bross, 2008; Santos et al., 2009; Smith, 2010; Theodore et al., 2007).

Diante do exposto, o presente trabalho descreveu a utilização do Child Neglect Index para identificar casos de crianças negligenciadas por seus pais/cuidadores no contexto brasileiro. Foi possível apresentar evidências empíricas relevantes sobre a utilidade e a sensibilidade desse recurso avaliativo no processo de identificação e caracterização da negligência para com crianças de seis a oito anos, principalmente para auxiliar profissionais que trabalham diretamente com essa faixa etária (como na área da Educação e Saúde). Embora sejam dados iniciais e de natureza exploratória sobre o Child Neglect Index (sendo necessária sua validação para o Brasil), obtidos em uma cidade do interior do Estado de São Paulo, mostram que esse instrumento poderá funcionar como recurso técnico auxiliar a profissionais que trabalham em órgãos de proteção das crianças e dos adolescentes (conselheiros tutelares, entre outros).

O instrumento mostrou-se capaz de confirmar os casos das crianças notificadas por negligência e as suspeitas de viverem essa problemática, porém não oficialmente notificadas. Assim, de modo geral, pode-se dizer que as crianças de G1 e de G2 apresentaram características que comporiam um quadro de negligência, que se materializou em indicadores adicionais à suspeita inicial de seus educadores no ambiente escolar. Isso deve ser considerado como um alerta, sendo a detecção, a notificação e o adequado manejo das situações de negligência infantil extremamente importante para que ocorram intervenções profissionais visando à diminuição das sequelas em seu desenvolvimento.

O estudo apresenta, no entanto, algumas limitações. Trata-se de trabalho inicial e de caráter exploratório a respeito das possibilidades informativas do Child Neglect Index para o Brasil. Considerou-se necessário divulgá-lo entre os profissionais da área, por se mostrar válido e preciso no diagnóstico da negligência infantil em outros países, como já apontado, porém exige ainda aprimoramentos técnico-científicos para uso em larga escala no contexto nacional, estímulo presentemente proposto nesse trabalho, favorecendo novas investigações. Cabe lembrar que o Child Neglect Index foi desenvolvido no contexto internacional, de modo que sua utilização no Brasil deve ser realizada com muitos cuidados, exigindo estudos adicionais de validação, entre outros aprimoramentos, também 
aqui sugeridos. Há ainda que se destacar que o número de casos presentemente avaliados, embora numericamente suficiente para as análises estatísticas aqui delineadas, pode ser considerado reduzido frente à complexidade do fenômeno, fazendo-se necessário ampliar e diversificar os indivíduos em análise.

Outra variável a ser aprimorada, ainda do ponto de vista técnico, é relativo ao processo de coleta de dados em si do Child Neglect Index. No caso do atual estudo apenas uma pesquisadora avaliou as díades e o conjunto de informações a respeito das crianças e de seus cuidadores. Seria bastante enriquecedor se esses registros e avaliações dos casos fossem realizados por avaliadores independentes, em processos simultâneos, ou por avaliadores treinados, porém "cegos" em relação à variável critério, na base da formação dos grupos em estudo. Isso ofereceria maior fidedignidade e sustentabilidade aos próprios achados desse instrumento de avaliação psicológica, trabalho por ser efetivado em estudo futuro.

Cabe, no entanto, referendar, com os atuais achados empíricos, a utilidade de instrumentos de avaliação psicológica para a detecção de complexos fenômenos e fatores de risco ao desenvolvimento infantil, como no caso do Child Neglect Index. O reduzido conhecimento de profissionais que trabalham com crianças (sobretudo no campo da Educação e da Saúde) a respeito dos maus-tratos a crianças, em especial do tipo da negligência, poderá ser adequadamente enfrentado na medida em que novos recursos técnicos estejam disponíveis e adequadamente balizados como auxiliares aos processos de atuação profissional cotidiana, motivador maior do presente trabalho. Certamente faz-se ainda necessário que o potencial diagnóstico e a validade do Child Neglect Index sejam adequadamente verificados em outras regiões do Brasil, dialogando com estudos em curso a partir de outros instrumentos avaliativos, também conduzidos com o objetivo do aprimoramento técnico-científico da área.

\section{Referências}

Ávila de Mello, I. L. M. (2008). Negligência de crianças: Contribuições para a avaliação de fatores de risco psicossociais (Dissertação de mestrado não-publicada, Universidade São Paulo, Ribeirão Preto, SP, Brasil).

Bazon, M. R. (2006). Estudo da prevalência de maus-tratos em crianças de 0 a 10 anos, frequentando estabelecimentos educacionais públicos e privados, e identificação de fatores de risco pessoais e sociais e de indicadores comportamentais e emocionais (Deliberação CONDECA/SP N. 02/05, Artigo 7).

Bazon, M. R. (2008). Violências contra crianças e adolescentes: Análise de quatro anos de notificações feitas no Conselho Tutelar na cidade de Ribeirão Preto, São Paulo, Brasil. Cadernos de Saúde Pública, 24(2), 323-332. doi:10.1590/ S0102-311X2008000200011

Bazon, M. R., Mello, I. L. M. A., Bérgamo, L. P. D., \& Faleiros, J. M. (2010). Negligência infantil: Estudo comparativo do nível sócio-econômico, estresse parental e apoio social. Temas em Psicologia, 18, 71-84.
Bérgamo, L. P. D. (2007). Maus-tratos fisicos de crianças: Contribuições para a avaliação de fatores de risco psicossociais (Dissertação de mestrado, Faculdade de Filosofia, Ciências e Letras de Ribeirão Preto, Universidade de São Paulo, Ribeirão Preto, SP, Brasil). Recuperado em http://www.teses.usp.br/ teses/disponiveis/59/59137/tde-07012009-222425/pt-br.php

Bérgamo, L. P. D., Pasian, S. R., Ávila de Mello, I. L. M., \& Bazon, M. R. (2009). O Inventário de Potencial de Maus-tratos Infantil: Estudo de precisão e validade. Avaliação Psicológica, 8, 425-435.

Bringiotti, M. I. (1999). Maltrato infantil. Madrid, España: Mino Dávila.

Bringiotti, M. I. (2000). La escuela ante los niños maltratados. Buenos Aires, Argentina: Paidós.

Brousseau, M. (2000). La perceptiondu fonctionnement familial de parents négligents e non négligents e le facteurs familiaux, parentaux et environnementaux associes. Québec, Canada: Faculté dês Sciences Sociales, École de Service Social, Université Laval.

Calheiros, M., \& Monteiro, M. B. (2000). Mau trato e negligência parental: Contributos para a definição social dos conceitos. Sociologia, Problemas e Práticas, 34, 145-176.

Corso, P. S., \& Fertig, A. R. (2010). The economic impact of child maltreatment in the United States: Are the estimates credible? Child Abuse Neglect, 34(5), 296-304.

Dubowitz, H. (2007). Understanding and addressing the "neglect of neglect": Digging into the molehill. Child Abuse \& Neglect, 31, 603-606. doi:10.1016/j.chiabu.2007.04.002

Dubowitz, H., Pitts, S. C., \& Black, M. (2004). Measurement of three major subtypes of child neglect. Child Maltreatment, 9(4), 344-356. doi:10.1177/1077559504269191

Fallon, B., Trocme, N., Fluke, J., MacLaurin, B., Tonmyr, L., \& Yuan, Y. (2010). Methodological challenges in measuring child maltreatment. Child Abuse \& Neglect, 34(1), 70-79. doi:10.1016/j.chiabu.2009.08.008

Ferreira, A. L. (2005). Acompanhamento de crianças vítimas de violência: Desafios para o pediatra. Jornal de Pediatria, 81(5), 173-180. doi:10.1590/S0021-75572005000700007

Glaser, D. (2002). Emotional abuse and neglect (psychological maltreatment): A conceptual framework. Child Abuse \& Neglect, 26, 697-714. doi:10.1016/S0145-2134(02)00342-3

Gonçalves, H. S., Ferreira, A. L., \& Marques, M. J. V. (2002). Avaliação de serviço de atenção a crianças vítimas de violência doméstica. Revista de Saúde Pública, 33(6), 47-53. doi:10.1590/S0034-89101999000600005

Gracia, E. (1995). Visible but unreported: A case for the "not serious enough" cases of child maltreatment. Child Abuse \& Neglect, 19, 1083-1093. doi:0145-2134(95)00070-4

Granville-Garcia, A. F., Souza, M. G. C., Menezes, V. A., Barbosa, R. G., \& Cavalcanti, A. L. (2009). Conhecimentos e percepção de professores sobre maus-tratos em crianças e adolescentes. Saúde e Sociedade, 18(1), 131-140.

Hahm, H. C., Lee, Y., Ozonoff, A., \& Van Wert, M. J. (2010). The impact of multiple types of child maltreatment on subsequent risk behaviors among women during the transition from adolescence to young adulthood. Journal of Youth Adolescence, 39, 528-540. doi:10.1007/s10964-009-9490-0

Lacharité, C., Éthier, L., \& Nolin, P. (2006). Vers une théorie écosystémique de la négligence envers les enfants. Bulletin de Psychologie, 59(4), 381-394. doi:10.3917/bupsy.484.0381

Lavergne, C., \& Tourigny, M. (2000). Incidence de l'abus et la négligence envers lês enfants: recension dês écrits. Criminologie, 33(1), 47-72. doi:10.7202/004730ar 
Lei no 8.069. (1990, 16 jul.). Dispõe sobre o Estatuto da Criança e do Adolescente e dá outras providências. Diário Oficial da União, Seção 1, p. 13563.

Martins, C. B. G. (2010). Maus tratos contra crianças e adolescentes. Revista Brasileira de Enfermagem, 63(4), 660-665.

Martins, F. F. S. (2006). Crianças negligenciadas: A face (in) visivel da violência familiar (Dissertação de mestrado, Pontifícia Universidade Católica de Minas Gerais, Belo Horizonte, MG, Brasil). Recuperada em http://www1.pucminas.br/ documentos/dissertacoes_fernanda_martins.pdf

Mathews, B., \& Bross, D. C. (2008). Mandated reporting is still a policy with reason: Empirical evidence and philosophical grounds. Child Abuse \& Neglect, 32(5), 511-516. doi:10.1016/j.chiabu.2007.06.010

Mayer, M., Lavergne, C., Tourigny, M., \& Wright, J. (2007). Characteristics differentiating neglected children from other reported children. Journal of Family Violence, 22(8), 721-732. doi:10.1007/s10896-007-9120-0

McSherry, D. (2007). Understanding and addressing the "neglect of neglect": Why are we making a mole-hill out of a mountain? Child Abuse \& Neglect, 31, 607-614. doi:10.1016/j. chiabu.2006.08.011

Milner, J. S. (1986). The child abuse potential inventory: Manual ( $2^{\text {nd }}$ ed.). Webster, TX: Psytec.

Nolin, P., \& Turgeon, M. (2004). Relation entre la négligence et la capacité de mémorisation et d'apprentissage verbal chez les enfants. Revue Québécoise de Psychologie, 25(2), 151-165.

O'Donnell, M., Scott, D., \& Stanley, F. (2008). Child abuse and neglect-is it time for a public health approach? Australian New Zealand Journal of Public Health, 32(4), 325-330. doi:10.1111/j.1753-6405.2008.00249.x.

Pasian, M. S. (2012) Maus-tratos infantis: O impacto da negligência no desenvolvimento psicossocial e acadêmico de crianças em fase inicial de escolarização (Tese de doutorado, Faculdade de Filosofia, Ciências e Letras de Ribeirão Preto, Universidade de São Paulo, Ribeirão Preto, SP, Brasil). Recuperada em http://www.ffclrp.usp.br/imagens_defesas/06_08_2012_14_10_11_61.pdf

Pires, A. L. D., \& Miyazaki, M. C. (2005). Maus-tratos contra crianças e adolescentes: Revisão da literatura para profissionais da saúde. Arquivos de Ciências da Saúde, 12(1), 42-49.

Reppold, C. T., Pacheco, J., Bardagi, M., \& Hutz, C. S. (2002). Prevenção de problemas de comportamento e desenvolvimento de competências psicossociais em crianças e adolescentes: Uma análise das práticas educativas e dos estilos parentais. In C. S. Hutz (Ed.), Situações de risco e vulnerabilidade na infância e na adolescência: Aspectos teóricos e estratégias de intervenção (pp. 7-51). São Paulo, SP: Casa do Psicólogo.

Rios, K. S. A. (2010). Inventário de potencial de abuso infantil - CAP: Adaptação transcultural, fidedignidade e validade para o Brasil (Tese de doutorado, Universidade Federal de São Carlos, SP, Brasil). Recuperada em http://www.laprev. ufscar.br/documentos/arquivos/teses-e-dissertacoes/tese-karyne-de-souza-augusto-rios.pdf

Rios, K. S. A., Williams, L. C. A., Schelini, P. W., Bazon, M. R., \& Piñón, E. A. (2013). Inventário de Potencial de Abuso Infantil - CAP: Evidências de validade e precisão. Avaliação Psicológica, 12(1), 51-60.

Roque, E. M. S. T., \& Ferriani, M. G. C. (2007). Estudo das famílias de crianças e adolescentes, vítimas de violência, que sofreram intervenção da justiça. Revista Latino-Americana de Enfermagem, 15(4), 334-344. doi:10.1590/S010411692007000400005
Santos, S. R., Ferreira, A. L., Paixão, A. C., Pfeiffer, L. Y., Aquino, L. A., \& Amaral, J. J. F. (2009). Adaptação e aplicabilidade do componente "maus-tratos" à estratégia da Atenção Integrada às Doenças Prevalentes na Infância no Brasil. Revista Brasileira Saúde Materno Infantil, 9(3), 359366. doi:10.1590/S1519-38292009000300014

Silva, M. A. I., \& Ferriani, M. G. C. (2007). Violência doméstica: Do visível ao invisível. Revista Latino-Americana de Enfermagem, 15(2), 275-281. doi:10.1590/S010411692007000200013

Silvany, C. M. S., Siqueira, I. C., Rolim, A. M., \& Cavalcante, S. S. (2011). Sinais de maus tratos em crianças e adolescentes hospitalizados por causas diversas em um hospital de referência em Salvador. Revista Baiana de Pediatria, 5(1), 27-33.

Slack, K. S., Holl, J., McDaniel, M., Yoo, J., \& Bolger, K. (2004). Understanding the risks of child neglect: An exploration of poverty and parenting characteristics. Child Maltreatment, 9(4), 395-408. doi:10.1177/1077559504269193

Smith, M. C. (2010). Early childhood educators: Perspectives on maltreatment and mandated reporting. Children and Youth Services Review, 32, 20-27.

Strauss, M. A., \& Kantor, G. K. (2005). Definition and measurement of neglectful behavior: Some principles and guidelines. Child Abuse \& Neglect, 29, 19-29. doi:10.1016/j. chiabu.2004.08.005

Sullivan, S. (2000). Child neglect: Current definitions and models a review of child neglect research, 1993-1998. Ottawa, Canada: National Clearinghouse on Family Violence.

Theodore, A., Runyan, D., \& Chang, J. J. (2007). Measuring the risk of physical neglect in a population-based sample. Child Maltreatment, 12(1), 96-105. doi:10.1177/1077559506296904

Trocmé, N. (1996). Development and preliminary evaluation of the Ontario Child Neglect Index. Child Maltreatment, 1(2), 145-155. doi:10.1177/1077559596001002006

World Health Organization, \& International Society for Prevention of Child Abuse and Neglect. (2006). Preventing child maltreatment: A guide to taking action and generating evidence. Retrieved December 10, 2011, from http://www. ispcan.org/publications.htm

Zuravin, S. J. (1999). Child neglect: A review of definitions and measurement research. In H. Dubowitz (Ed.), Neglected children: Research, practice and policy (pp. 156-173). Thousand Oaks, CA: Sage. 